\title{
Abnormal Auditory Mismatch Fields in Children and Adolescents with 47,XYY Syndrome
}

\author{
Junko Matsuzaki ${ }^{a} \quad$ Luke Bloy $^{a} \quad$ Lisa Blaskey ${ }^{a, b} \quad$ Judith Miller $^{b} \quad$ Emily S. Kuschner ${ }^{a, b}$ \\ Matthew Ku ${ }^{a}$ Marissa Dipiero ${ }^{a}$ Megan Airey ${ }^{\mathrm{a}} \quad$ J. Christopher Edgar ${ }^{\mathrm{a}}$ David Embick $^{\mathrm{c}}$ \\ Judith L. Ross ${ }^{\text {d, e }}$ Timothy P.L. Roberts ${ }^{a, c}$ \\ a Lurie Family Foundations MEG Imaging Center, Department of Radiology, Children's Hospital of Philadelphia, \\ Philadelphia, PA, USA; ${ }^{b}$ Center for Autism Research, Children's Hospital of Philadelphia, Philadelphia, PA, USA; \\ 'Department of Linguistics, University of Pennsylvania, Philadelphia, PA, USA; ${ }^{d}$ Thomas Jefferson University,

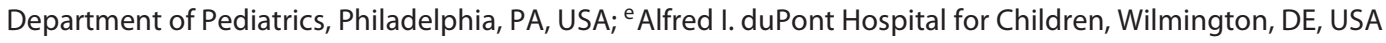

\section{Keywords}

XYY syndrome - Autism spectrum disorder .

Magnetoencephalography · Vowel mismatch fields .

Language impairment

\begin{abstract}
$47, \mathrm{XYY}$ syndrome (XYY) is one of the common forms of sex chromosome aneuploidy in males. XYY males tend to have tall stature, early speech, motor delays, social and behavioral challenges, and a high rate of language impairment. Recent studies indicate that $20-40 \%$ of males with XYY meet diagnostic criteria for autism spectrum disorder (ASD; the rate in the general population is $1-2 \%$ ). Although many studies have examined the neural correlates of language impairment in ASD, few similar studies have been conducted on individuals with XYY. Studies using magnetoencephalography (MEG) in idiopathic ASD (ASD-I) have demonstrated delayed neurophysiological responses to changes in the auditory stream, revealed in the mismatch negativity or its magnetic counterpart, the mismatch field (MMF). This study investigated whether similar findings are observed in XYYassociated ASD and whether delayed processing is also present in individuals with XYY without ASD. MEG measured
\end{abstract}

MMFs arising from the left and the right superior temporal gyrus during an auditory oddball paradigm with vowel stimuli $(/ a /$ and $/ \mathrm{u} /$ ) in children/adolescents with XYY both with and without a diagnosis of ASD, as well as in those with ASDI and in typically developing controls (TD). Ninety male participants (6-17 years old) were included in the final analyses (TD, $n=38,11.50 \pm 2.88$ years; ASD-I, $n=21,13.83 \pm 3.25$ years; $X Y Y$ without ASD, $n=15,12.65 \pm 3.91$ years; $X Y Y$ with ASD, $n=16,12.62 \pm 3.19$ years). The groups did not differ significantly in age $(p>0.05)$. There was a main effect of group on MMF latency $(p<0.001)$. Delayed MMF latencies were found in participants with XYY both with and without an ASD diagnosis, as well as in the ASD-I group compared to the TD group ( $p s<0.001)$. Furthermore, participants with XYY (with and without ASD) showed a longer MMF latency than the ASD-I group ( $p s<0.001$ ). There was, however, no significant difference in MMF latency between individuals with XYY with ASD and those with XYY without ASD. Delayed MMF latencies were associated with severity of language impairment. Our findings suggest that auditory MMF latency delays are pronounced in this specific $Y$ chromosome aneuploidy disorder, both with and without an ASD diagnosis, and thus may implicate the genes of the $Y$ chromosome in mediating atypical MMF activity.

\section{KARGER}

(c) 2019 S. Karger AG, Basel

E-Mail karger@karger.com

www.karger.com/dne
Timothy P.L. Roberts, PhD

Oberkircher Family Chair in Pediatric Radiology and Vice-Chair Research Department of Radiology, Children's Hospital of Philadelphia

3401 Civic Center Blvd, Philadelphia, PA 19104 (USA)

E-Mail robertstim@email.chop.edu 


\section{Introduction}

47 , XYY syndrome (hereafter, XYY) is one of the common sex chromosome aneuploidy/sex chromosome trisomy conditions and occurs in 1 in 1,000 males [1-3]. The cognitive phenotype in boys with XYY typically includes normal to mildly diminished general intelligence $[4,5]$, with the verbal IQ commonly more impaired than the performance IQ [6-8]. Most boys with XYY are diagnosed postnatally in the first decade of life because of developmental delays, behavioral issues, tall stature [5], autistic behaviors $[9,10]$, and language impairments [11-14].

In a neuroanatomical study on boys with XYY, Bryant et al. [15] assessed global and regional brain matter variations associated with XYY. The authors suggested that the genetic variations associated with XYY were related to an increased brain volume, a finding putatively linked to the increased frequency of autism spectrum disorder (ASD) among individuals with XYY, with rates of ASD in XYY of up to $20-40 \%[8,9,13,14]$.

Brain functional studies (i.e., electroencephalography and magnetoencephalography [MEG] studies) have reported abnormal auditory language discrimination processing (i.e., delayed latency) in ASD [16-18], assessed by the mismatch negativity (MMN) potential or its magnetic counterpart, the mismatch field (MMF). Such findings have been reported for infants, children, and adults with ASD $[17,19]$, which suggests a persistence of the processing deficit. The MMN/MMF reflects the ability of an individual to detect changes in the auditory stream and thus is thought to index neural discrimination processing [1624]. The ability to detect an auditory change (e.g., pitch, frequency, and syllable) is an important neural processing feature, and compromise thereof likely contributes to downstream language and communication impairment in individuals with developmental disorders such as ASD $[16,17]$.

In an early MMN/MMF ASD study, Tecchio et al. [22] reported that moderately to severely verbally impaired individuals with ASD (8-32 years old) demonstrated a weak or absent MMF response compared with typically developing controls (TD; tone burst oddball paradigm). They suggested that an impaired auditory discrimination ability in preconscious cortical processing stages may hinder the development of more complex connections in ASD. Oram Cardy et al. [18] reported on MMFs elicited by changes in streams of vowels or spectrally matched tones in children and adolescents with ASD and TD (8-17 years old) to explore whether impaired sound discrimination may contribute to language impairments in ASD. Al- though MMFs were observed in both groups, the children with ASD demonstrated a delayed MMF latency compared with the TD. The authors suggested that difficulty parsing transient differences in sounds may lead to impaired acoustic or phonological representations and subsequent language impairment in ASD. Berman et al. [23] showed an association between MMF latency delay and language impairment in children with ASD (8-12 years old), as well as a corresponding relationship to microstructural diffusion tensor MRI of the arcuate fasciculus, supporting a white matter connectivity disruption hypothesis for the biological basis of MMF delays. While these studies discuss the latency of the MMF component in ASD, a recent study by Matsuzaki et al. [19] reported an atypical hemispheric bias in MMF amplitude (tending rightward in adults with ASD, compared to leftward in TD). The functional significance of this asymmetry is yet to be determined. Although many MMF studies have been conducted on ASD populations [21,22], investigations of the neural correlates of auditory and language processing in children and adolescents with XYY are very limited [25].

To contribute to the knowledge of the neurophysiological mechanisms of auditory language discrimination processes in children and adolescents with XYY, MEG was used to measure cortical responses to an auditory oddball paradigm with vowel stimuli (/a/ and /u/) identical to those used by Roberts et al. [16], and compared with age-matched children with idiopathic (no known genetic etiology) ASD (ASD-I) as well as with TD. Hypotheses were: (1) MMF latencies arising from the superior temporal gyrus (STG) would be delayed in children and adolescents with XYY with and without ASD compared to those with ASD-I and TD; (2) MMF latency delays may be exacerbated in participants with XYY with ASD compared to those with XYY without ASD; (3) delayed MMF latencies would be associated with language ability; and (4) atypical rightward lateralization of MMF amplitudes would be evidenced in both children and adolescents within the ASD-I group and both XYY groups.

\section{Subjects and Methods}

Participants: Recruitment and Inclusion/Exclusion Criteria Males with a karyotypic diagnosis of nonmosaic XYY (6-17 years old) were recruited through the eXtraordinarY Kids Clinics (for children with sex chromosome variations) at Nemours/Alfred I. duPont Hospital for Children. Age- and gender-matched (male) individuals with ASD as well as TD were recruited from the Children's Hospital of Philadelphia (CHOP) via CHOP's Recruitment Enhancement 
Core, which enables recruitment of children seen anywhere within the CHOP care system (e.g., primary and specialty care centers and specialty practices). The XY status (in either the ASD-I or the TD cohort) was not confirmed by genetic testing; with a 1/1,000 XYY prevalence, the expected number of undiagnosed XYY subjects inadvertently included in our XY subcohort total of 59 subjects is 0.06 , and inadvertent inclusion is thus considered unlikely.

The participants in the TD group had no current or past history of DSM-5 axis I disorders according to parent report during initial screening and had no symptoms of ASD (i.e., were below the diagnostic cutoffs), as measured by direct observation on the Autism Diagnostic Observation Schedule 2nd edition (ADOS-2) [26], Social Communication Questionnaire (SCQ) [27], and Social Responsiveness Scale-2 (SRS-2) [28]. The children in the ASD-I group had a prior diagnosis of ASD, made by autism specialists at CHOP or other regional hospitals and practices, according to DSM-5 criteria and with utilization of autism diagnostic tools. The current diagnostic battery confirmed the original diagnosis in the ASD-I group via the ADOS-2, SCQ, and SRS-2. Given the complexity of establishing an ASD diagnosis in the XYY cohort, the diagnosis in the XYY+ASD group was established by expert clinical consensus between two clinical psychologists (J. Miller and L. Blaskey), who reviewed all available diagnostic information and case conferenced each participant to arrive at a consensus diagnosis.

Dimensional symptom severity indices were obtained from the ADOS-2 Calibrated Severity Score metric [29] and from the SRS-2 [28]. A measure of full-scale IQ (FSIQ) was obtained for all participants. The specific measure of FSIQ utilized varied depending on group and included both the DAS-II [30] and the WISC-V [31]. Language function was assessed with the Clinical Evaluation of Language Fundamentals-5th Edition (CELF-5) [32].

The following inclusion/exclusion criteria were used for all participants: (1) no history of traumatic brain injury or other significant medical or neurological abnormality, or other genetic syndrome; (2) no active psychosis; (3) no MRI contraindications; (4) no significant sensory impairments (e.g., vision or hearing impairment); (5) English as the first language; and (6) no known drug or alcohol use prior to any study procedure. Additionally, all participants were required to score at or above the 2 nd percentile (SS >70) on an index of nonverbal IQ via the WISC-V or DAS-II. Nine children were prescribed stimulant medications at the time of participation. The data from these participants did not show evidence of forming an outlier cluster in terms of MMF responses, and therefore these data were retained. Data from some TD participants $(n=18)$ have in part been previously submitted for publication and are included herein to provide context for the XYY responses.

\section{Auditory Stimuli}

The auditory vowel stimuli $(/ \mathrm{a} /$ and $/ \mathrm{u} /)$ were presented using E-Prime v1.1 experimental software (Psychology Software Tools Inc., Pittsburgh, PA, USA). They were delivered via a sound pressure transducer and sound conduction tubing to the subject's peripheral auditory canal via ear tip inserts (ER-3A; Etymotic Research, Elk Grove Village, IL, USA) at $45 \mathrm{~dB}$ sensation level; 300$\mathrm{ms}$ stimuli (vowels $/ \mathrm{a} /$ and $/ \mathrm{u} /$ ) were played with each token as the standard (85\%) or the deviant (15\%) stimulus and with a stimulus onset asynchrony of $700 \mathrm{~ms}$ [16]. Two runs with the vowels alternating as standard/deviant were conducted to allow matched token subtraction (i.e., deviant /a/ - standard /a/ and deviant /u/ standard $/ \mathrm{u} /$ ).

Abnormal MMFs in Children and

Adolescents with XYY Syndrome

\section{MEG Recording}

MEG data were recorded in a magnetically shielded room using a 275-channel whole-cortex CTF magnetometer (CTF MEG, Coquitlam, BC, Canada). At the start of the session, three head position indicator coils were attached to the scalp to provide continuous specification of the position and orientation of the head relative to the MEG sensor array [16]. Foam wedges were inserted inside of the MEG dewar by the side of each subject's head to increase the subject's comfort and ensure that the head remained in the same location within the dewar across recording sessions. To minimize fatigue and encourage an awake state, the subjects viewed (but did not listen to) a movie projected on to a screen positioned at a comfortable viewing distance. To identify eyeblink activity, the electro-oculogram (EOG; bipolar oblique, upper right and lower left sites) was collected. Electrodes were also attached to the left and right collarbones for electrocardiographic recording.

\section{Data Analysis}

Analyses were performed blinded to participant group. As previously described [19], after a band-pass filter $(0.03-150 \mathrm{~Hz})$, the EOG, electrocardiography, and MEG signals were downsampled to $500 \mathrm{~Hz}$. Synthetic third-order gradiometer environmental noise reduction was implemented for the MEG data. Epochs of $600 \mathrm{~ms}$ duration ( $100 \mathrm{~ms}$ before the stimulus to $500 \mathrm{~ms}$ after the stimulus) were defined from the continuous recording.

To remove eyeblink-related artifacts, a typical eyeblink was manually identified in the raw data (including EOG) for each participant. The pattern search function in BESA Research 6.1 (BESA GmbH, Muldestausee, Germany) was then used to scan the raw data to identify other similar blink events and to compute an eyeblink average. An eyeblink was modeled by its first component topography from principal component analysis, typically accounting for more than $99 \%$ of the variance in the eyeblink average. In addition to eyeblink activity, a heartbeat average was obtained, and activity was modeled by the first two principal component analysis component topographies of a heartbeat average, typically accounting for more than $85 \%$ of the variance in the heartbeat average. Reviewing the eyeblink- and heartbeat-corrected raw data, epochs with artifacts other than blinks and heartbeats were rejected by amplitude and gradient criteria (amplitude $>300 \mathrm{fT}$, gradients $>25 \mathrm{fT} / \mathrm{cm}$ ).

Using all 275 channels of the MEG data, determination of the latency and amplitude of sources of the auditory evoked field in the bilateral STG was accomplished by applying a standard source model which transformed each individual's raw MEG recordings into brain space (MEG data coregistered to the Montreal Neurologic Institute [MNI-152] averaged brain) using a model with multiple sources [33-35]. Specifically, the standard source model applied to each subject was constructed by including (1) bilateral STG dipole sources (placed at Heschl's gyrus), and (2) nine fixed regional sources that modeled brain background activity and served as probe sources for additional electrophysiological activity. The eyeblink and heartbeat source vectors derived for each participant were also included in each participant's source model to account for eyeblink and heartbeat activity [36, 37]. The final source model served as a montage for the raw MEG data [38, 39]. This spatial filter thus disentangled the source activities of the different brain regions that had overlapped at the sensor level. For the source analysis, a $1-\mathrm{Hz}$ (12 dB/octave, zero-phase) to $55-\mathrm{Hz}(48$ $\mathrm{dB} /$ octave, zero-phase) band-pass filter and a powerline notch filter at $60 \mathrm{~Hz}$ (width $5 \mathrm{~Hz}$ ) were applied. 
Table 1. Characteristics of the study participants

\begin{tabular}{lcccc}
\hline & TD & ASD-I & XYY-ASD & XYY+ASD \\
\hline Participants, $n$ & 38 & 21 & 15 & 16 \\
Handedness (R:L:A), $n$ & $31: 7: 0$ & $21: 0: 0$ & $15: 0: 0$ & $13: 2: 1$ \\
Age, years & $11.50 \pm 2.88$ & $13.83 \pm 3.25$ & $12.65 \pm 3.91$ & $12.62 \pm 3.19$ \\
SCQ score & $2.36 \pm 2.40$ & $18.20 \pm 5.97$ & $9.87 \pm 6.37$ & $13.56 \pm 5.87$ \\
SRS score & $41.51 \pm 7.85$ & $73.10 \pm 11.45$ & $70.47 \pm 16.80$ & $76.31 \pm 16.16$ \\
GAI/estimated FSIQ/GCA score & $113.58 \pm 14.41$ & $106.50 \pm 17.48$ & $92.00 \pm 11.03$ & $87.00 \pm 16.10$ \\
CELF core language score & $107.46 \pm 11.78$ & $97.95 \pm 17.47$ & $90.20 \pm 15.58$ & $78.07 \pm 13.40$ \\
\hline
\end{tabular}

Values are presented as mean \pm SD unless specified otherwise. TD, typically developing children; ASD, autism spectrum disorder; ASD-I, idiopathic ASD; XYY-ASD, 47,XYY syndrome without ASD; XYY+ASD, 47,XYY syndrome with ASD; SCQ, Social Communication Questionnaire; SRS, Social Responsiveness Scale; GAI/ estimated FSIQ/GCA, General Ability Index/estimated full-scale intelligence quotient/General Conceptual Ability score (GAI and estimated FSIQ: Wechsler Intelligence Scale - 4th edition [WISC-IV] and 5th edition [WISC-V]; GCA: Differential Ability Scale - II [DAS-II]); CELF core language score, Clinical Evaluation of Language Fundamentals - 4th and 5th editions.

MMF peaks were defined from the difference wave obtained by subtraction of the response to the standard token from the response deviant for each token, with the MMF peak identified as the first peak following the residual 100 -ms response in the subtracted waveform (and occurring 150-350 ms after stimulus onset). The difference wave was obtained separately for standard and deviant occurrences of each token from source-space waveforms. MMF responses were thus defined for each hemisphere and each token separately. To evaluate hemispheric laterality, laterality indices (LIs) were computed for each token using the formula

$$
L I=\frac{(L H-R H)}{(L H+R H)},
$$

where $\mathrm{LH}$ and $\mathrm{RH}$ represent the amplitude of the MMF in the left and the right hemisphere, respectively.

\section{Statistics}

Potential effects of group (TD, ASD-I, XYY-ASD, XYY+ASD) on age and neuropsychological assessments were evaluated with analysis of variance. Potential effects of group (TD, ASD-I, $\mathrm{XYY}-\mathrm{ASD}, \mathrm{XYY}+\mathrm{ASD})$, hemisphere $(\mathrm{LH}, \mathrm{RH})$, and token $(/ \mathrm{a} /$ and $/ \mathrm{u} /$ ) on MMF latency and amplitude were evaluated with full factorial linear mixed models (LMMs) using these factors as fixed effects and age as a covariate with subject as a random effect. LIs of MMF amplitudes were assessed using an LMM with group and stimulus token as fixed effects, age as a covariate, and subject as a random effect. Hierarchical regression assessed the influence of language and general cognitive ability above and beyond effects of age, hemisphere, and stimulus token on MMF latency and amplitude. For the LIs, hierarchical regression also assessed the influence of language and cognitive ability above and beyond effects of age and stimulus token. Bonferroni correction was applied for multiple comparisons. All statistical analyses were performed with SPSS Statistics version 25 (IBM, Armonk, NY, USA).

\section{Results}

\section{Demographics}

Twenty-three participants who did not meet the neuropsychological assessment criteria for inclusion, or who did not complete all measurements or did not have analyzable MEG data, were excluded from analysis. As shown in Table 1, 90 male participants (aged 6-17 years) remained and were included in the final analyses (TD, $n=38,11.50 \pm 2.88$ years; ASD-I, $n=21,13.83 \pm 3.25$ years; $\mathrm{XYY}-\mathrm{ASD}, n=15,12.65 \pm 3.91$ years; $\mathrm{XYY}+\mathrm{ASD}$, $n=16,12.62 \pm 3.19$ years). The groups did not differ significantly in age $(p>0.05)$. There were statistically significant main effects of group on SCQ score $(F(3,83)=$ $50.81, p<0.001)$, SRS-2 $(F(3,84)=48.91, p<0.001)$, CELF core language score $(F(3,81)=16.29, p<0.001)$, and General Ability Index/FSIQ $(F(3,83)=15.07, p<$ $0.001)$. The post hoc tests revealed differences between TD and all groups, as well as between the ASD-I group and both XYY groups, in SCQ score ( $p s<0.05)$. There were group differences between the TD group and the other three groups in SRS-2 score $(p s<0.05)$. There were differences between the TD group and both XYY groups, as well as between the ASD-I group and both XYY groups, in FSIQ ( $p s<0.05)$. There were differences between the TD group and both XYY groups, as well as between the ASD-I group and the XYY+ASD (but not the XYY-ASD) group in CELF core language index $(p s<0.05)$. There were no differences between the $\mathrm{XYY}-\mathrm{ASD}$ group and the XYY+ASD group in SCQ score, SRS-2 score, FSIQ, or CELF score. 
Fig. 1. Source response waveforms from the superior temporal gyrus. The vertical line on the waveform indicates stimulus onset $(0 \mathrm{~ms})$. The arrow indicates mismatch field (MMF) latency in a representative typically developing child (168 ms). The solid gray line indicates the standard response, the dashed gray line indicates the deviant response, and the solid black line indicates their subtraction to yield the difference wave.

Fig. 2. Estimated marginal mean mismatch field (MMF) latencies by group and hemisphere. Error bars represent 1 standard error of the marginal means. There was a significant main effect of group on MMF latency $(p<0.0001)$, which was evidenced in both hemispheres. Post hoc $t$ tests revealed significant differences between pairwise comparisons of all groups (except $\mathrm{XYY}+\mathrm{ASD}$ vs. XYY-ASD), in both hemispheres (** all $p s<0.001$ ), with XYY+ASD and XYY-ASD > ASD-I > TD. TD, typically developing controls; ASD-I, idiopathic autism spectrum disorder; XYY-ASD, 47,XYY syndrome without ASD; XYY+ ASD, 47,XYY syndrome with ASD.
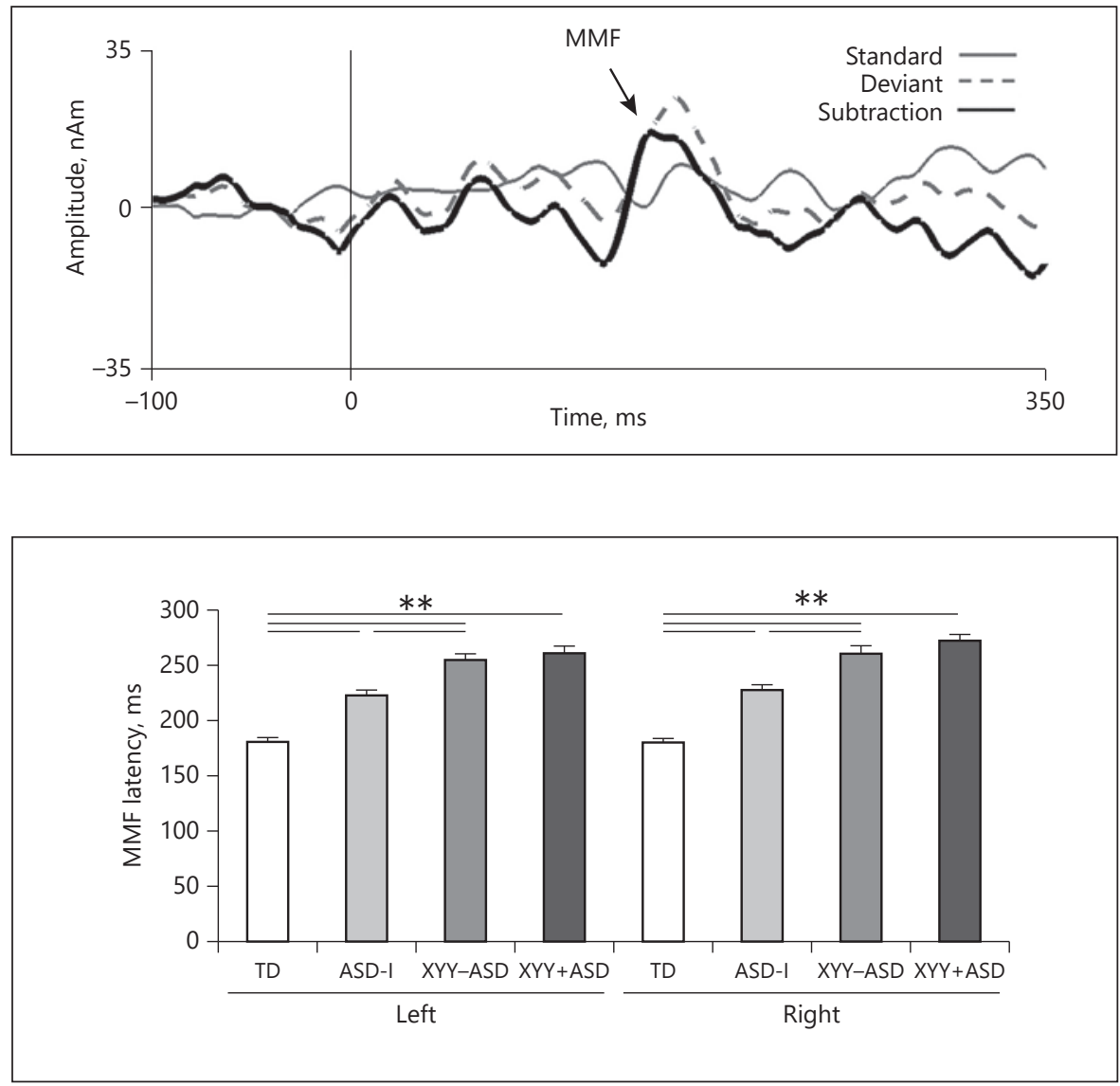

\section{MMF Latencies and Amplitudes}

Sample MMF waveforms are shown in Figure 1. An LMM with fixed effects of group, hemisphere, and stimulus token and age (and with subject as a random effect) showed significant effects of group (TD, $180.75 \pm 3.57$ ms; ASD-I, $225.62 \pm 4.83 \mathrm{~ms}$; XYY-ASD, $258.82 \pm 5.84$ $\mathrm{ms}$; XYY+ASD, $267.80 \pm 5.24 \mathrm{~ms} ; F(3,76)=82.85, p<$ $0.001)$ and hemisphere $(\mathrm{LH}, 230.61 \pm 2.58 \mathrm{~ms}$; RH, $235.73 \pm 2.58 \mathrm{~ms} ; F(1,228)=11.12, p<0.001)$ and a small effect of stimulus token $(/ \mathrm{a} /, 231.59 \pm 2.58 \mathrm{~ms} ; / \mathrm{u} /$, $234.76 \pm 2.58 \mathrm{~ms} ; F(1,228)=4.24, p<0.05)$ on MMF latency, and no interactions ( $p$ s $>0.05$ ). Delayed MMF latencies were found in both hemispheres in participants with XYY both with and without ASD, as well as in the ASD-I group, compared to the TD group ( $p s<$ 0.001 ; Fig. 2). The delays in the XYY group (with and without ASD) were pronounced compared to those in the ASD-I group $(p<0.001)$. However, there were no group differences between individuals with XYY with ASD and those with XYY without ASD $(p>0.05)$. There was no significant effect of age and no age $\times$ group interaction.

Abnormal MMFs in Children and

Adolescents with XYY Syndrome
Hierarchical regression was used to investigate the association between MMF latency and language ability across the whole cohort: when entered first (after age, hemisphere, and stimulus token had accounted for $R^{2}=$ $0.05)$, FSIQ accounted for significant variance $\left(\Delta R^{2}=0.24\right.$, $p<0.001)$ and CELF core language score accounted for additional significant variance $\left(\Delta R^{2}=0.07, p<0.001\right)$ in MMF latency. When the order was reversed, CELF score $\left(\Delta R^{2}=0.30, p<0.001\right)$ and FSIQ $\left(\Delta R^{2}=0.017, p<0.01\right)$ again both accounted for significant variance in MMF latency, suggesting unique contributions from both FSIQ and CELF score to MMF latency, despite FSIQ and CELF score themselves being correlated $(R=0.74, p<0.001)$.

An LMM with fixed effects of group, hemisphere, and stimulus token and age (and with subject as a random effect) showed a significant effect of group on MMF amplitude (TD, $13.05 \pm 2.03 \mathrm{nAm}$; ASD-I, $19.44 \pm 2.59$ nAm; XYY-ASD, $8.34 \pm 3.35$ nAm; XYY+ASD, $10.14 \pm$ $2.93 \mathrm{nAm} ; F(3,72)=2.99, p<0.05)$, with no significant effect of hemisphere $(\mathrm{LH}, 13.40 \pm 1.61 \mathrm{nAm}$; RH, $12.08 \pm 1.61 \mathrm{nAm} ; F(1,216)=0.63, p>0.05)$ or stimulus token $(/ \mathrm{a} /, 13.62 \pm 1.61 \mathrm{nAm} ; / \mathrm{u} /, 11.87 \pm 1.61 \mathrm{nAm} ; F(1$, 
$216)=0.29, p>0.05)$ and no interactions $(p s>0.05)$. The ASD-I group tended to show an increased MMF amplitude compared to the TD group, but there were no group differences by post hoc $t$ test $(p>0.05)$. There were no associations of MMF amplitude with FSIQ or CELF core language score.

\section{Laterality Indices}

An LMM with fixed effects of group and stimulus token and age (and with subject as a random effect) showed a significant effect of group on LIs (TD, $+0.06 \pm 0.04$; ASD-I, $-0.09 \pm 0.06$; XYY $-\mathrm{ASD},-0.23 \pm 0.07$; XYY + ASD,$-0.15 \pm$ $0.06 ; F(3,144)=3.96, p<0.01)$, with no significant effect of stimulus token $(/ \mathrm{a} /,-0.12 \pm 0.04 ; / \mathrm{u} /,-0.06 \pm 0.04 ; F(1$, $144)=0.81, p>0.05)$ and no interactions ( $p s>0.05)$. While the TD group showed leftward lateralization, the ASD-I group and both XYY groups showed atypical rightward lateralization. The post hoc tests revealed differences between the TD group and either XYY group (TD vs. XYYASD, $p<0.05$; TD vs. XYY+ASD, $p=0.059$ ). The differences between the TD group and the ASD-I group did not reach significance $(p>0.05)$. Regarding the association between LI and FSIQ/CELF score, when entered first (after age and stimulus token had accounted for $R^{2}=0.09$ ), FSIQ $\left(\Delta R^{2}=0.026, p<0.05\right)$ and then (when entered second) CELF core language score $\left(\Delta R^{2}=0.023, p<0.05\right)$ both accounted for significant variance in LIs. When the order was reversed, whereas CELF score did not account for significant variance $\left(\Delta R^{2}=0.000, p>0.05\right)$, when entered next, FSIQ accounted for significant variance in LIs $\left(\Delta R^{2}=0.05\right.$, $p<0.01$ ), suggesting the LI was sensitive to general cognitive ability and not language ability in this cohort.

\section{Discussion}

The main findings of this study were that (1) there were delayed MMF latencies in XYY children with and without ASD compared with children with ASD or TD; (2) there were no differences in the extent of the delay between the XYY+ASD group and the XYY-ASD group; (3) delayed latencies were associated with poorer performance on language and IQ tests; and (4) there was a lack of neurotypical left-hemisphere lateralization of the MMF amplitude in XYY children (with and without ASD).

Previous studies have reported prolonged MMF latencies in higher-functioning children and adults with ASD $[16,19,40]$. For example, using a similar oddball paradigm with vowel stimuli, Roberts et al. [16] reported that auditory MMF abnormalities are frequently observed in children with ASD aged 6-15 years, and that MMF abnormalities may have sequelae in terms of clinical language impairment. In particular, MMF delays were most pronounced $(\sim 50 \mathrm{~ms})$ in children with ASD with concomitant language impairments, suggesting a neurobiological basis as well as a clinical biomarker for language impairment in ASD. In an adult ASD cohort using a similar auditory vowel MMF task, Matsuzaki et al. [19] found that the MMF was delayed in adults with ASD compared to the TD participants ( $\sim 15 \mathrm{~ms})$. Furthermore, whereas the TD participants showed a leftward lateralization of the MMF amplitude, the participants with ASD showed a rightward lateralization. The findings suggest that adults with ASD have hemispherically and temporally abnormal auditory discrimination processing.

The present study found a delayed MMF latency in ASD-I compared to TD children and adolescents ( $\sim 45$ $\mathrm{ms}$ ), a finding consistent with previous ASD studies. Furthermore, individuals with XYY (with and without a diagnosis of ASD) showed a delayed MMF latency even compared to ASD-I subjects ( 30-40 ms). Previous studies have suggested that MMF/MMN latency decreases as individuals mature $[41,42]$. In this study, the XYY participants had normal to mildly diminished general intelligence and language ability (XYY-ASD, FSIQ $92.00 \pm$ 11.03, CELF score $90.20 \pm 15.58$; XYY+ASD, FSIQ $87.00 \pm 16.10$, CELF score $78.07 \pm 13.40$ ). We speculate that XYY children (with and without ASD) may have delayed white matter myelination and atypical connectivity for both bottom-up and top-down attentional/inhibitory control networks compared to the relatively higher-functioning children with ASD (ASD-I, FSIQ $106.50 \pm 17.48$, CELF score $97.95 \pm 17.47$ ) and TD.

The findings of atypical rightward MMF amplitude lateralization are consistent with MEG studies on children and adults with ASD $[19,43]$. Hemisphere differences in MMF amplitude may be interpreted in light of differences in the developmental trajectories of each hemisphere, and in ASD the reversed lateralization is possibly associated with dysfunction of interhemispheric inhibition and/or connectivity. Lin et al. [44] reported on individuals with sex chromosome aneuploidy, suggesting that the $\mathrm{X}$ and $\mathrm{Y}$ chromosomes may have opposing effects on cortical development and cortical thickness asymmetry $[45,46]$. Further, they suggested these patterns might be related to reported differences in cognitive profile and interhemispheric connectivity in sex differences [47]. Atypical laterality may also be considered in terms of the potential effects of early testosterone exposure, as discussed by Geschwind and Galaburda [48]. 
Interhemispheric inhibition is also dependent on the microstructure of the corpus callosum (CC), which connects homologous cortical areas of the two cerebral hemispheres. In several ASD studies, using diffusion-weighted MRI, decreased fractional anisotropy and increased mean diffusivity in white matter tracts spanning the CC have been reported $[49,50]$, suggesting impaired interhemispheric connectivity. Whereas in the present study the children and adolescents with XYY did not show any differences in laterality compared to the ASD-I group, the abnormalities relative to the TD group may be related to both autistic features and/or cognitive ability. Further studies are needed to examine associations between LI and fractional anisotropy and mean diffusivity in white matter tracts spanning the CC in XYY and ASD cohorts.

Additionally, previous MEG research has also revealed abnormal gamma band oscillatory activity during taskevoked auditory processing in ASD [51-54]. Wilson et al. [51] examined the integrity of the local circuitry by focusing on the gamma band activity of auditory magnetic steady-state responses, and the study reported that the production and/or maintenance of left-hemispheric gamma oscillations appeared abnormal in children and adolescents with ASD, suggesting that a lack of local inhibitory interneurons may be the neural mechanism underlying this impairment due to the known role of such cells in generating high-frequency activity. Edgar et al. [53] assessed activity in STG auditory areas in children with ASD and reported decreased early evoked gamma activity and inter-trial coherence in children with ASD. Port et al. [54] reported perturbed auditory cortex neural activity during sinusoidal tones, as evidenced by M100 latency delays as well as reduced transient gamma band activity in children with ASD.

It has been proposed that ASD may be associated, at least in a subset of the population, with imbalances in excitatory and inhibitory neurotransmission (E/I imbalance) [55]; gamma band activity is thought to be one neural process related to $\mathrm{E} / \mathrm{I}$ balance, with support coming from observed correlations between gamma and underlying GABA concentrations [e.g., 56]. This hypothesis would be attractive in that it implicates a biochemical target for intervention, and it might represent an objective marker of ASD. It has thus motivated concomitant investigation of GABA, primarily using magnetic resonance spectroscopy, as part of a multimodal characterization of ASD, work that could readily be extended to XYY. In general, it is of clear and immediate interest to observe whether the range of electrophysiological (and neurochemical) anomalies that have been reported in children and ado-

Abnormal MMFs in Children and Adolescents with XYY Syndrome lescents with ASD (including both evoked responses and oscillatory activity) are recapitulated in XYY (as is shown for the MMF findings reported in the present study).

Finally, the observation of a delayed MMF in XYY with ASD and XYY without ASD may point to a role of the genes of the $\mathrm{Y}$ chromosome in mediating brain responses. It is of note that in the general population, the prevalence of ASD is reported as being 4 times greater among males than among females (i.e., approx. 2.5\%) [57]. The rate of ASD in XYY is reported as being as high as $20-40 \%$ [8, 9, $13,14]$., and those individuals with XYY who do not meet the strict diagnostic criteria for ASD may still manifest behavioral and clinical symptoms associated with ASD $[9,10]$ (note the elevated SRS scores and diminished FSIQ and language function in this cohort). If we interpret the MMF delay as an indicator of diminished capacity in general cognitive ability or domain-specific language ability, it is not surprising that the XYY-ASD cohort exhibited a delayed MMF response (consistent with their clinical/ behavioral phenotype) despite the fact that they did not meet the diagnostic criteria for ASD per se.

A potential study limitation is that there were no individuals recruited with other sex chromosome aneuploidy/sex chromosome trisomy conditions (i.e., XXY/Klinefelter syndrome and XXYY). Further, the precise relation to the findings from the literature on ASD-I is tempered by our inclusion of only male subjects (to gender-match to the XYY cohort), while many other studies on ASD-I include at least some female participants. Also, the apparent association between MMF latency and language ability could be consistent with theories that auditory language discrimination and acquisition of vowel stimuli are related to either basic sensory functions or higher-level language processes. To examine the dependencies between lower-order sensory perception and higher-order linguistics skills, other tasks, using word, pseudoword, or sentence listening, may provide more detailed information regarding MMF and language impairment. Additionally, we did not assess other modalities (e.g., cortical myelin content, diffusion tensor imaging, and GABA magnetic resonance spectroscopy) and so cannot address any structural or neurochemical underpinnings of the observed MMF response delays.

\section{Conclusions}

This study demonstrated a delayed MMF in children and adolescents with XYY and showed that delayed MMF responses were associated with greater language impair- 
ment and lower IQ. Although the MMF latencies were also delayed in ASD-I, the MMF latency delays were more profound in XYY. There was no additional delay in XYY individuals who met the diagnostic criteria for ASD versus those who did not, perhaps suggesting a XYY "ceiling effect." The findings support a neurophysiological signature of language impairment in XYY similar to that seen in ASD, and thus provides some shared evidence for considering XYY as a human genetic model of at least a subset of ASD.

\section{Acknowledgements}

T.P.L. Roberts and the authors gratefully acknowledge all participants, their families, and the Oberkircher family for the Oberkircher Family Chair in Pediatric Radiology. Excellent assistance was provided by John Dell, Rachel Golembski, Peter Lam, and the MEG laboratory team of the Department of Radiology at CHOP.

\section{Statement of Ethics}

The study was approved by the CHOP Internal Review Board and by the Human Studies Committee at Nemours/Alfred I. duPont Hospital for Children. Written informed consent and assent (when age appropriate) was obtained from all participating families, in accordance with the principles of the Declaration of Helsinki.

\section{Disclosure Statement}

T.P.L. Roberts declares consulting/advisory board relationships with Prism Clinical Imaging, CTF, Ricoh, Spago Nanomedical, AveXis Inc., and Acadia Pharmaceuticals. Additionally, T.P.L. Roberts and J.C. Edgar disclose intellectual property related to MEG as a biomarker for pharmaceutical therapy, under licensing negotiation.

\section{Funding Sources}

This study was supported in part by NIH R21-MH109158 (T.P.L. Roberts/J.L. Ross), NIH R01-DC008871 (T.P.L. Roberts), R01-HD073258 (D. Embick), and U54-HD086984 (Neuroimaging Core: T.P.L. Roberts), as well as by a grant from the Department of Defense (AR140927; T.P.L. Roberts/J.L. Ross).

\section{References}

1 Jacobs PA, Melville M, Ratcliffe S, Keay AJ, Syme J. A cytogenetic survey of 11,680 newborn infants. Ann Hum Genet. 1974 May; 37(4):359-76.

2 Nielsen J, Sillesen I. Incidence of chromosome aberrations among 11,148 newborn children. Humangenetik. 1975 Oct;30(1):112.

3 Leggett V, Jacobs P, Nation K, Scerif G, Bishop DV. Neurocognitive outcomes of individuals with a sex chromosome trisomy: XXX, XYY, or XXY: a systematic review. Dev Med Child Neurol. 2010 Feb;52(2):119-29.

4 Bender BG, Puck MH, Salbenblatt JA, Robinson A. The development of four unselected 47,XYY boys. Clin Genet. 1984 May;25(5): $435-45$.

5 Robinson A, Bender BG, Puck MH, Salbenblatt JA. Growth and Development of Children With a 47,XYY Karyotype. New York (NY): Alan R. Liss, Inc; 1985.

6 Christensen AL, Nielsen J. Psychological studies of ten patients with the XYY syndrome. Br J Psychiatry. 1973 Aug;123(573): 219-21.

7 Ratcliffe SG, Field MA. Emotional disorder in XYY children: four case reports. J Child Psychol Psychiatry. 1982 Oct;23(4):401-6.

8 Ross JL, Roeltgen DP, Kushner H, Zinn AR, Reiss A, Bardsley MZ, et al. Behavioral and social phenotypes in boys with 47,XYY syndrome or 47,XXY Klinefelter syndrome. Pediatrics. 2012 Apr;129(4):769-78.
9 Bishop DV, Jacobs PA, Lachlan K, Wellesley D, Barnicoat A, Boyd PA, et al. Autism, language and communication in children with sex chromosome trisomies. Arch Dis Child. 2011 Oct;96(10):954-9.

10 American Psychiatric Association. Diagnostic and Statistical Manual of Mental Disorders, 5th Edition. Washington (DC): American Psychiatric Publishing, Inc.; 2013.

11 Lehrke RG. Birth defects original article series. Ann Intern Med. 1971;74(1):157.

12 Merhar SL, Manning-Courtney P. Two boys with 47, XXY and autism. J Autism Dev Disord. 2007 May;37(5):840-6.

13 Tartaglia NR, Wilson R, Miller JS, Rafalko J, Cordeiro L, Davis S, et al. Autism Spectrum Disorder in Males with Sex Chromosome Aneuploidy: XXY/Klinefelter Syndrome, XYY, and XXYY. J Dev Behav Pediatr. 2017 Apr; 38(3):197-207.

14 van Rijn S. A review of neurocognitive functioning and risk for psychopathology in sex chromosome trisomy (47,XXY, 47,XXX, 47, XYY). Curr Opin Psychiatry. 2019 Mar;32(2):79-84.

15 Bryant DM, Hoeft F, Lai S, Lackey J, Roeltgen D, Ross J, et al. Sex chromosomes and the brain: a study of neuroanatomy in XYY syndrome. Dev Med Child Neurol. 2012 Dec;54(12):1149-56.

16 Roberts TP, Cannon KM, Tavabi K, Blaskey L, Khan SY, Monroe JF, et al. Auditory magnetic mismatch field latency: a biomarker for language impairment in autism. Biol Psychiatry. 2011 Aug;70(3):263-9.
17 Schwartz S, Shinn-Cunningham B, Tager-Flusberg $\mathrm{H}$. Meta-analysis and systematic review of the literature characterizing auditory mismatch negativity in individuals with autism. Neurosci Biobehav Rev. 2018 Apr;87:106-17.

18 Oram Cardy JE, Flagg EJ, Roberts W, Roberts TP. Delayed mismatch field for speech and non-speech sounds in children with autism. Neuroreport. 2005 Apr;16(5):521-5.

19 Matsuzaki J, Ku M, Berman JI, Blaskey L, Bloy $\mathrm{L}$, Chen YH, et al. Abnormal auditory mismatch fields in adults with autism spectrum disorder. Neurosci Lett. 2019 Apr;698:140-5.

20 Näätänen R, Gaillard AW, Mäntysalo S. Early selective-attention effect on evoked potential reinterpreted. Acta Psychol (Amst). 1978 Jul; 42(4):313-29.

21 Näätänen R, Paavilainen P, Rinne T, Alho K. The mismatch negativity (MMN) in basic research of central auditory processing: a review. Clin Neurophysiol. 2007 Dec;118(12): 2544-90.

22 Tecchio F, Benassi F, Zappasodi F, Gialloreti LE, Palermo M, Seri S, et al. Auditory sensory processing in autism: a magnetoencephalographic study. Biol Psychiatry. 2003 Sep; 54(6):647-54.

23 Berman JI, Edgar JC, Blaskey L, Kuschner ES, Levy SE, Ku M, et al. Multimodal DiffusionMRI and MEG Assessment of Auditory and Language System Development in Autism Spectrum Disorder. Front Neuroanat. 2016 Mar;10:30. 
24 Yoshimura Y, Kikuchi M, Hayashi N, Hiraishi $\mathrm{H}$, Hasegawa C, Takahashi T, et al. Altered human voice processing in the frontal cortex and a developmental language delay in 3- to 5 -year-old children with autism spectrum disorder. Sci Rep. 2017 Dec;7(1):17116.

25 Bloy L, Ku M, Edgar JC, Miller JS, Blaskey L, Ross J, et al. Auditory evoked response delays in children with 47,XYY syndrome. Neuroreport. 2019 May;30(7):504-9.

26 Lord C, Rutter M, DiLavore PC, Risi S, Gotham K, Bishop SL. Autism Diagnostic Observation Schedule. 2nd ed. Torrance (CA) Western Psychological Services; 2012.

27 Rutter M, Bailey A, Lord C. The Social Communication Questionnaire: Manual. Los Angeles (CA): Western Psychological Services; 2003.

28 Constantino JN, Davis SA, Todd RD Schindler MK, Gross MM, Brophy SL, et al. Validation of a brief quantitative measure of autistic traits: comparison of the Social Responsiveness Scale with the Autism Diagnostic Interview-Revised. J Autism Dev Disord. 2003 Aug;33(4):427-33.

29 Gotham K, Pickles A, Lord C. Standardizing ADOS scores for a measure of severity in autism spectrum disorders. J Autism Dev Disord. 2009 May;39(5):693-705.

30 Elliott CD. Differential Ability Scales. 2nd ed San Antonio (TX): Harcourt Assessment; 2007.

31 Wechsler D. Wechsler Intelligence Scale for Children. 5th ed. San Antonio (TX): Pearson; 2014.

32 Wiig EH, Semel E, Secord WA. Clinical Evaluation of Language Fundamentals - Fifth Edition (CELF-5). Pearson, San Antonio, TX. 2013.

33 Scherg M, von Cramon D. A new interpretation of the generators of BAEP waves I-V: results of a spatio-temporal dipole model. Electroencephalogr Clin Neurophysiol. $1985 \mathrm{Jul}$ 62(4):290-9.

34 Scherg M. Fundamentals of dipole source potential analysis. Adv audiol. 6th ed. Basel, Switzerland: Karger; 1990. pp. 40-69.

35 Scherg M, Berg P. New concepts of brain source imaging and localization. Electroencephalogr Clin Neurophysiol Suppl. 1996;46: $127-37$.
36 Lins OG, Picton TW, Berg P, Scherg M. Ocular artifacts in recording EEGs and event-related potentials. II: source dipoles and source components. Brain Topogr. 1993;6(1):65-78.

37 Berg P, Scherg M. A multiple source approach to the correction of eye artifacts. Electroencephalogr Clin Neurophysiol. 1994 Mar; 90(3):229-41.

38 Scherg M, Ebersole JS. Brain source imaging of focal and multifocal epileptiform EEG activity. Neurophysiol Clin. 1994 Jan;24(1):51-60.

39 Scherg M, Ille N, Bornfleth H, Berg P. Advanced tools for digital EEG review: virtual source montages, whole-head mapping, correlation, and phase analysis. J Clin Neurophysiol. 2002 Apr;19(2):91-112.

40 Roberts TP, Heiken K, Kahn SY, Qasmieh S, Blaskey L, Solot C, et al. Delayed magnetic mismatch negativity field, but not auditory M100 response, in specific language impairment. Neuroreport. 2012 May;23(8):463-8.

41 Glass E, Sachse S, von Suchodoletz W. Auditory sensory memory in 2-year-old children: an event-related potential study. Neuroreport. 2008 Mar;19(5):569-73.

42 Morr ML, Shafer VL, Kreuzer JA, Kurtzberg D. Maturation of mismatch negativity in typically developing infants and preschool children. Ear Hear. 2002 Apr;23(2):118-36.

43 Flagg EJ, Cardy JE, Roberts W, Roberts TP. Language lateralization development in children with autism: insights from the late field magnetoencephalogram. Neurosci Lett. 2005 Sep;386(2):82-7.

44 Lin A, Clasen L, Lee NR, Wallace GL, Lalonde F, Blumenthal J, et al. Mapping the stability of human brain asymmetry across five sex-chromosome aneuploidies. J Neurosci. 2015 Jan; 35(1):140-5.

45 Herbert MR, Harris GJ, Adrien KT, Ziegler DA, Makris N, Kennedy DN, et al. Abnormal asymmetry in language association cortex in autism. Ann Neurol. 2002 Nov;52(5):588-96.

46 Shaw P, Kabani NJ, Lerch JP, Eckstrand K, Lenroot R, Gogtay N, et al. Neurodevelopmental trajectories of the human cerebral cortex. J Neurosci. 2008 Apr;28(14):3586-94.

47 Ingalhalikar M, Smith A, Parker D, Satterthwaite TD, Elliott MA, Ruparel K, et al. Sex differences in the structural connectome of the human brain. Proc Natl Acad Sci USA. 2014 Jan;111(2):823-8.
48 Geschwind N, Galaburda AM. Cerebral lateralization. Biological mechanisms, associations, and pathology: I. A hypothesis and a program for research. Arch Neurol. 1985 May;42(5):428-59.

49 Travers BG, Adluru N, Ennis C, Tromp PM, Destiche D, Doran S, et al. Diffusion tensor imaging in autism spectrum disorder: a review. Autism Res. 2012 Oct;5(5):289-313.

50 Giuliano A, Saviozzi I, Brambilla P, Muratori F, Retico A, Calderoni S. The effect of age, sex and clinical features on the volume of Corpus Callosum in pre-schoolers with Autism Spectrum Disorder: a case-control study. Eur J Neurosci. 2018 Mar;47(6):568-78.

51 Wilson TW, Rojas DC, Reite ML, Teale PD, Rogers SJ. Children and adolescents with autism exhibit reduced MEG steady-state gamma responses. Biol Psychiatry. 2007 Aug; 62(3):192-7.

52 Rojas DC, Wilson LB. $\gamma$-Band abnormalities as markers of autism spectrum disorders. Biomarkers Med. 2014;8(3):353-68.

53 Edgar JC, Fisk CL IV, Berman JI, Chudnovskaya D, Liu S, Pandey J, et al. Auditory encoding abnormalities in children with autism spectrum disorder suggest delayed development of auditory cortex. Mol Autism. 2015 Dec;6(69):69.

54 Port RG, Edgar JC, Ku M, Bloy L, Murray R, Blaskey L, et al. Maturation of auditory neural processes in autism spectrum disorder - a longitudinal MEG study. Neuroimage Clin. 2016 Apr;11:566-77.

55 Rubenstein JL, Merzenich MM. Model of autism: increased ratio of excitation/inhibition in key neural systems. Genes Brain Behav. 2003 Oct;2(5):255-67.

56 Port RG, Gaetz W, Bloy L, Wang DJ, Blaskey L, Kuschner ES, et al. Exploring the relationship between cortical GABA concentrations, auditory gamma-band responses and development in ASD: evidence for an altered maturational trajectory in ASD. Autism Res. 2017 Apr;10(4):593-607.

57 Ozonoff S, Young GS, Carter A, Messinger D, Yirmiya N, Zwaigenbaum L, et al. Recurrence risk for autism spectrum disorders: a Baby Siblings Research Consortium study. Pediatrics. 2011 Sep;128(3):e488-95. 